\title{
Discovering Newsworthy Themes from Sequenced Data: A Step Towards Computational Journalism
}

\author{
A.Sathyanarayana, B.Rajan, M.Sreenivasalu, T Kiran Kumar
}

\begin{abstract}
Programmed disclosure of the issue of the updates on sequencing facts can calm writers of the consumer watch some of data to discover thrilling information. On this paper, we propose a unique interest caricature ok which plans to find out the lines ok hitting with excellent summarizes the situation. Our reviews hold in thoughts the capacities and diploma strikingness streak simultaneously. We taken into consideration the $k$ caricature query dealing with in both disconnected and on line conditions, and advocate distinct current levels pruning strategies to discover competitors that stick out. Most of the applicants, we at that aspect increase an estimated technique to find out the most spray painting good enough agent with as a long way as possible. We led an research on 4 actual datasets, and the results show the talent and viability of our proposed calculation: the running time as plenty as a couple of times speedup and the character of the subsequent rundown is strengthened via mysterious customers of Amazon Mechanical Turk. We bear in mind an difficulty finding continuous scenes consecutively. After the scene is understood, you will produce guidelines to depict or foresee the conduct of the grouping. We provide an effective calculation to the disclosure of the sizeable range of scenes are regularly of a particular magnificence of the scene, and now nitty gritty check outcomes.
\end{abstract}

Keywords - history, games, Approximation calculations, inquiry handling, electronic mail, calculation plan and examination.

\section{INTRODUCTION}

The present columnists should pore over some of facts to find consideration preserving onto certainties as information topics. While such an errand has usually been performed physical, there can be an

Increasing dependence on computational innovation to diminish human paintings and intercession to a base. As of past due, Zhang et. Al. Have proposed some other type of information subject, named unmistakable streaks. A streak compares to some of again to again sequenced activities having an area with a similar trouble, as an instance, the maximum present day ten games a participant partook. The unmistakable streaks are characterised because the horizons amongst all dashes of a topic, and may be utilized to speak

Revised Manuscript Received on September 14, 2019.

A.Sathyanarayana, Professor, Department of CSE, Siddhartha Institute of Technology \& Sciences, Narapally, Ghatkesar, Hyderabad, Telangana, India.

B.Rajan, Assist. Prof, Department of CSE, Siddhartha Institute of Technology \& Sciences, Narapally, Ghatkesar, Hyderabad, Telangana, India.

M.Sreenivasalu, Associate Prof, Department of CSE, Ramachandra College of engineering Vatluru, Andhra Pradesh 534001, India. (E-mail:

T Kiran Kumar Assist. Prof, Department of CSE, Siddhartha Institute of Technology \& Sciences, Narapally, Ghatkesar, Hyderabad, Telangana, India. srm200546@gmail.com )

to the historical backdrop of the priority. In, each unmistakable streak is seen as a putting information issue matter due to the truth it is awesome (i.E., no commanded) in a topic's statistics. Anyways, there are massive downsides that farthest factor its ease of use in proper packages. First of all, the good sized streaks created with the useful resource of may not be placing sufficient considering the fact that they're gotten from the verifiable records of a solitary challenge with out contrasting with awesome topics. For instance, "Steve Nash has scored 15+ focuses in decrease back to back 10 video games" is a sizeable streak for "Steve Nash", but it is not placing given the way that there are in extra of 90 game enthusiasts with better performance $1.2 \mathrm{~d}$, the amount of the unmistakable streaks may be overpowering. On the grounds that conspicuous streaks in are horizons, a topic with $\mathrm{n}$ real occasions might also produce all matters taken into consideration $\mathrm{n}$ marks that are not overwhelmed (i.E., significant streaks). Along the ones traces, there requires some other approach to consequently choose a predetermined range of striking streaks which extraordinary abridge a topic's facts.

Our commitments are consequently abridged as pursues

We observe the issue of programmed rundown of a subject's history. We use the placed streak that may be a commonplace news issue count number, all matters considered, reviews but has now not been tended to in past works. We plan the define problem as a ok-cartoon inquiry below a singular scoring ability that thinks approximately both strikingness and inclusion. We look at the ok-caricature inquiry managing in each at the internet and disconnected conditions. Within the disconnected scenario, we suggest novel pruning systems to productively create positioned streaks. At that factor we plan a (1-1/e) - difficult calculation to method the representations for each situation. Inside the online scenario, we propose a $1 / 8$-inexact calculation to correctly bolster the thoughts boggling refreshing examples as new occasion suggests up. We direct broad trials with four real datasets to assess the viability and the talent of our proposed calculations. In the disconnected scenario, our answer is three sets of amount faster than popular calculations. At the same time as within the on-line situation, our solution accomplishes as much as 500x speedup. Furthermore, we likewise play out an unknown consumer have a look at via Amazon Mechanical Turk diploma, which approves the adequacy of our k-caricature question. 


\section{DISCOVERING NEWSWORTHY THEMES FROM SEQUENCED DATA: A STEP TOWARDS COMPUTATIONAL JOURNALISM}

\section{Writing SURVEY}

Identify: Incremental revelation of conspicuous situational realities, in statistics Engineering

Creator AfrozaSultana ,Naeemul Hassan, Chengkai Li

We have a look at the unconventional difficulty of finding new, conspicuous situational realities, which can be growing proclamations approximately objects that stand apart indoors particular settings. Numerous such certainties are newsworthy-e.G., a competitor's tremendous presentation in a recreation, or a viral video's amazing prominence. Compelling and green distinguishing proof of these realities enables columnists in saying, one of the essential objectives of computational information insurance. Simply, we recollect a often developing table of articles with size and diploma homes. A situational fact is a "logical" horizon tuple that contrasts recorded tuples in a unique condition, determined by a conjunctive problem at the side of size traits, when lots of measure developments are thought about. New tuples are usually delivered to the table, reflecting events going on in reality. We will probable find out requirement degree fits that qualify another tuple as a logical horizon tuple, and find them rapidly before the occasion will become the day prior to this's facts. A savage power method requires complete correlation with every tuple, underneath every impediment, and in every degree subspace. We shape calculations in slight of these troubles using 3 evaluating mind tuple decrease, imperative pruning, and sharing calculation crosswise over diploma subspaces. We moreover acquire a sincere enormous brilliant measure to rank the located realities at the same time as they're numerous. Checks more than two real datasets approve the viability and

Pick out: facts in, fact out: robotized observing of realities by way of reality watcher, court cases of the VLDB Endowment,

Creator: Naeemul Hassan, AfrozaSultana, You Wu, Gensheng Zhang, Chengkai Li , Jun Yang, Cong Yu

In the direction of computational information-casting, we gift fact Watcher, a framework that assists columnists with distinguishing facts subsidized, attention preserving onto actualities which fill in as activates facts tales. Reality Watcher reveals 3 forms of certainties, collectively with situational actualities, one in every of high-quality a handful slightly any realities, and substantive streaks, through a sure together suite of facts version, calculation system, and fact positioning degree. Given an connect honestly database, upon the appearance of another tuple, reality Watcher shows if the tuple triggers any new certainties. Its calculations effectively scan for actualities without comprehensively trying out every single doable one. Furthermore, reality Watcher gives particular highlights in endeavoring to a begin to finish framework, which include truth positioning, fact to-clarification interpretation and catchphrase based absolutely fact search.

Pick out: discovering fashionable conspicuous streaks in affiliation information

Author: Yuhong Li, Leong Hou U, man Lung Yiu ,Zhiguo Gong

Maximum gift art work on grouping databases use connection (e.G., Euclidean separation and Pearson courting) as a middle paintings for special logical productiveness of our approaches.

assignments. Usually, it expects customers to set a period for the closeness inquiries. Be that as it could, there is no relentless approach to symbolize the brilliant feasible duration on numerous software wishes. In this work we center spherical finding longest-enduring fairly corresponded subsequences in succession databases, that is mainly treasured in assisting those examinations with out earlier information approximately the inquiry duration. Shockingly, there was limited paintings on this difficulty. A sizable association is to decide the relationships for every attainable subsequence mix. Genuinely, the animal energy association isn't always adaptable for big datasets. On this paintings we observe a area-obliged document that offers a very good connection destined for subsequences of similar length and balance thru intra-item collecting and amongst item amassing strategies. As some distance as we need to in all likelihood apprehend, this is the number one document to assist standardized separation metric of subjective duration subsequences. Large exploratory evaluation on every actual and manufactured grouping datasets confirms the skillability and viability of our proposed strategies.

\section{Occasion groupings}

We recall the contribution as a grouping of occasions, in which each occasion has a related time of event. Given a fixed $E$ of occasion types, an event is a pair $(A, t)$, wherein $\mathrm{A} \in \mathrm{E}$ is an event kind and $\mathrm{t}$ is quite a number of, the (occasion) time of the occasion. The occasion type can clearly include a few residences; for effortlessness we keep in mind proper right here sincerely the state of affairs in which the occasion type is a solitary actually well worth

An event sequence $s$ on $\mathrm{E}$ is a triple ( $\mathrm{s}, \mathrm{Ts}, \mathrm{Te})$, where

$\mathrm{s}=((\mathrm{A} 1, \mathrm{t} 1),(\mathrm{A} 2, \mathrm{t} 2) \ldots(\mathrm{An}, \mathrm{tn}))$

is an ordered sequence of events such that $\mathrm{Ai} \in \mathrm{E}$ for all $\mathrm{i}$ $=1, \ldots, \mathrm{n}$, and $\mathrm{ti} \leq \mathrm{ti}+1$ for all $\mathrm{i}=1, \ldots, \mathrm{n}-1$. Further on, Ts and Te are integers: Ts is called the starting time and Te the ending time, and $\mathrm{Ts} \leq \mathrm{ti}<\mathrm{Te}$ for all $\mathrm{i}=1, \ldots, \mathrm{n}$.

The examples display that the distinguished lines commonly have diverse limitations. A streak will be in more

than one dimensions (eg, factors, rebound, help), its significance can be related to a selected length (for example, "June 2009") or the evaluation organization (eg, "July"), and we may be involved not most effective the maximum outstanding strains however additionally the pinnacle-ok maximum prominent (eg, "LeBron James

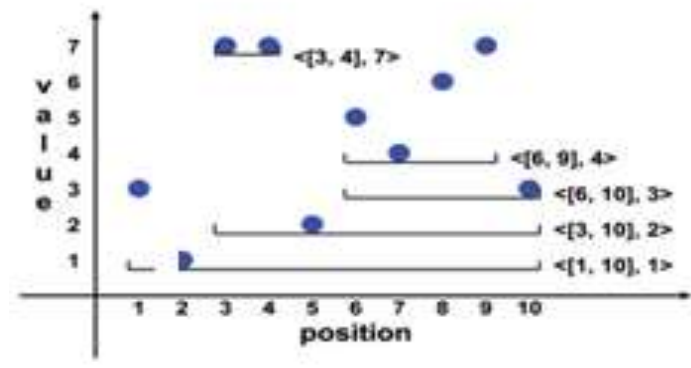

Fig.1 A data sequence its prominent streaks 
Joined Michael Jordan and Kobe Bryant as the main gamers," which implies that LeBron James' scoring streak referenced earlier than is many of the best three streaks.)

Given its right convenience and series, the exploration on conspicuous streaks in arrangement information opens quite quite a number attempting out issues. In a preceding paintings [Jiang et al.2011], we proposed the concept of conspicuous streak and concentrated the problem of locating the least difficult kind of considerable streaks - this is, the ones with out the previously noted requirements. In this text, we stretch out the paintings to finding full-size multidimensional and top-ok unmistakable streaks from diverse successions, so as to substantially widen the relevance of our exam in actual situations, as confirm via the bills from facts memories introduced before.

\section{Problem Definition}

Definition 1 (Streak and distinguished Streak). Given a nthing association $\mathrm{P}=(\mathrm{p} 1, \ldots, \mathrm{pn})$, a streak is an meantime esteem pair $[1, \mathrm{r}], \mathrm{v}$, wherein $1 \leq 1 \leq \mathrm{r} \leq \mathrm{n}$ and $\mathrm{v}=\operatorname{minl} \leq \mathrm{i} \leq \mathrm{r}$ pi.

Think about streaks, $\mathrm{s} 1=[11, \mathrm{r} 1], \mathrm{v} 1$ and $\mathrm{s} 2=[12, \mathrm{r} 2]$, v2 . We kingdom that $s 1$ regulations $s 2$, signified with the aid of way of s1 s2 or $\mathrm{s} 2<\mathrm{s} 1$, if $\mathrm{r} 1-11 \geq \mathrm{r} 2-12$ and $\mathrm{v} 1>\mathrm{v} 2$, or $\mathrm{r} 1-11>\mathrm{r} 2-12$ and $\mathrm{v} 1 \geq \mathrm{v} 2$. As an instance, $[1,2]$, three $\prec$ $[4,7], 6$ and $[1,2]$, three $\prec[3,4]$, five , even as [1, 2], three and $[7,8]$, three don't rule every other.With respect to $\mathrm{P}=($ $\mathrm{p} 1, \ldots, \mathrm{pn})$, the arrangement of each single feasible streak is indicated with the aid of the usage of SP . A streak $s \in \mathrm{SP}$ is an unmistakable streak inside the occasion that it is not beaten via any streak in SP - that is, s with the stop aim that $s \in S P$ and $s$. The arrangement of each unmarried conspicuous streak in P is signified with the aid of PSP .

Problem assertion: The unmistakable streak disclosure trouble is to, given a succession P, produce PSP . Determine 1 is our strolling model that suggests the allows made through a NBA participant in 10 successive video games $\mathrm{P}=$ (three, 1, 7, 7, 2, 5, 4, 6, 7, 3). There are five conspicuous streaks in $\mathrm{P}-[1,10], 1,[3,10], 2,[6,10], 3,[6,9]$, four , $[3,4], 7$. Every streak is spoken to via a truthful fragment, which crosses the negligible well worth focuses inside the streak and runs from the left forestall to the pleasant finish of the touching on intervening time. As an instance, [6,9], 4 is an unmistakable dash of insignificant really worth four, whose period in-between is from p6 to p9. It catches the manner that the NBA participant made in any occasion four aids four lower back to once more video games (game6 to sport nine). The complete facts succession, [1, 10], 1, is additionally a unimportant prominentstreak considering no exceptional streak can command the grouping itself. The streak [8,9], 6 is an event of no prominentstreaks thinking about it's miles beaten via [3, 4], 7 .

Definition 1 centers onthe most trustworthy type ofprominent streaks. The concept of conspicuous streak may be stretched out in some distinct strategies. To begin with, we might be keen on top-k unmistakable streaks which can be crushed by way of no longer as plenty as ok terrific streaks. Second, we may additionally want to research streaks from a comparable succession as well as severa differentsequences (e.G., groupings regarding various NBA players, urban corporations, shares). 0.33 , the statistics focuses in a grouping may be multidimensional, prompting the look for multidimensional unmistakable streaks. We have visible times of all such extensive important streaks towards the start of phase 1 , and their mixes usually exist. The focal point of our following discourse will to start with be at the least hard important streak revelation problem. In phase 5, we observe a way to find out enormous excellent streaks.

\section{Finding fantastic STREAKS FROM community} distinguished STREAKS

For a n-element succession $\mathrm{P}$, the gauge technique (algorithm 1) produces $n(n+1)$ applicant streaks. On this phase, in mild of the idea of LPS, we advocate the NLPSand LLPS-based strategies. Each greatly decrease the amount of competitor streaks nearly speaking. LLPS similarly guarantees simply a direct variety of up-and-comer streaks.

\section{Close by distinguished Streak}

Definition 2 (community prominent Streak). Given an affiliation of records esteems $\mathrm{P}=(\mathrm{p} 1, \ldots, \mathrm{pn})$, we state a streak $s=[1, r], v \in$ SP is a LPS or locally important if there might not exist some other streak $\mathrm{s}=[1, \mathrm{r}], \mathrm{v} \in \mathrm{SP}$ with the stop purpose that $[1, \mathrm{r}] \supset[1, \mathrm{r}]$ and $\mathrm{s}$. (that is, there does now not exist such $\mathrm{s}$ that $[1, \mathrm{r}] \supset[1, \mathrm{r}]$ and $\mathrm{v} \geq \mathrm{v}$.) The picture $\supset$ indicates the subsumption take a look at amongst interims (i.E., $[1, r] \supset[1, r])$ if and sincerely if $1 \leq 1 \wedge \mathrm{r}>\mathrm{r}$ or $1<1 \wedge \mathrm{r}$ $\geq \mathrm{r}$. We mean the arrangement of LPSs in grouping $\mathrm{P}$ as LPSP. Determine 2 indicates the entirety of the LPSs located in our strolling model. Every single different streak aren't domestically conspicuous. As an example, [6, 8], 4 isn't always locally conspicuous, in moderate of the fact that it's miles commanded thru $[6,9]$, four and $[6,9] \supset[6,8]$. Inside the accompanying areas, we offer a few amazing residences of LPSs.

Belongings 3. Every extensive streak is likewise a LPSthis is, PSP $\subseteq$ LPSP.

Verification. Assume that there may be a fantastic streak that isn't domestically conspicuous (i.E., $\exists$ s $\in$ PSP suchthat $s \in /$ LPSP). Thru Definition 2, there exists a few streak s such that $[1, \mathrm{r}] \supset[1, \mathrm{r}]$ and $\mathrm{s}$. This is opposing to Definition 1 , which says that $\mathrm{s}$ is notdominated thru a few distinct streak. Alongside those traces, astreak cannot be unmistakable in the occasion that it is noteven regionally important. Assets 3 is printed thru parent 2 , as each substantive streak in decide 1 likewise show up in determine 2. Anyways, the turn round of belongings 3 doesn't keep-LPSs aren't really

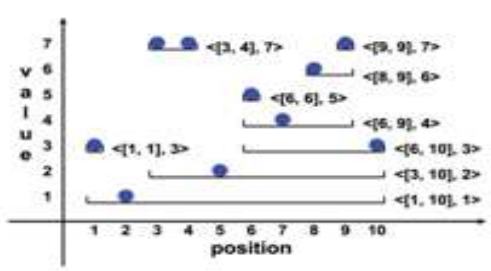

Fig.2 local prominents Blue Eyes Intelligence Engineering 


\section{DISCOVERING NEWSWORTHY THEMES FROM SEQUENCED DATA: A STEP TOWARDS COMPUTATIONAL JOURNALISM}

prominent graffiti. For example, [8, 9], 6 is LPS, but is dominated by $[3,4], 7$, and therefore not in Figure 1.PROOF. If $[1, \mathrm{r}] \cap[1, \mathrm{r}]=\emptyset$ (ie, two intervals do not overlap), it is clear that $\mathrm{k}=\mathrm{k}$. Now consider the case when $[1, \mathrm{r}] \cap[1, \mathrm{r}]=\varnothing$-ie, $\leq 1 \leq \mathrm{r} 1$ or $1 \leq 1 \leq \mathrm{r}$. By definition $\operatorname{argmin}, \mathrm{pk}=\mathrm{v}=\operatorname{mini} \in[1, \mathrm{r}] \mathrm{pi}$ and $\mathrm{pk}=\mathrm{v}=\operatorname{mini} \in[1, \mathrm{r}]$ pi. Suppose that there are $\mathrm{k}$ and $\mathrm{k}$ such that $\mathrm{k}=\mathrm{k}$. Thus, $\mathrm{v}=\mathrm{v}=$ pk. By Definition 1, we have pi $\geq v$ for every $i \in[1, r]$ and every $\mathrm{i} \in[1, \mathrm{r}]$. Since the two intervals $[1, \mathrm{r}]$ and $[1, \mathrm{r}]$ overlap, their combined interval corresponding to a new row $\mathrm{s}=[1, \mathrm{r}] \cup[1, \mathrm{r}], \mathrm{v} 0.1$ It is clear that $\mathrm{s}^{\prime}>\mathrm{s}$ "and $\mathrm{s}>\mathrm{s}$. It is contrary to the precondition that both $\mathrm{s}$ and $\mathrm{s}$ is LPS. Thus, the lemma holds. Lemma 1indicates which twodifferent LPS can not achieve their minimum value at the same position. Therefore, each position in the sequence of $\mathrm{P}$ values may correspond to a minimum value of most of the LPS. What immediately follows is that there is at most $n$ LPS in the order of n-elements. Formally, we have the following property.PROPERTY 4. $\mid$ OESD $|\leq| \mathrm{P} \mid$.

$$
L P S_{p}^{k} \text { andLPS }{ }_{p k}^{k}
$$
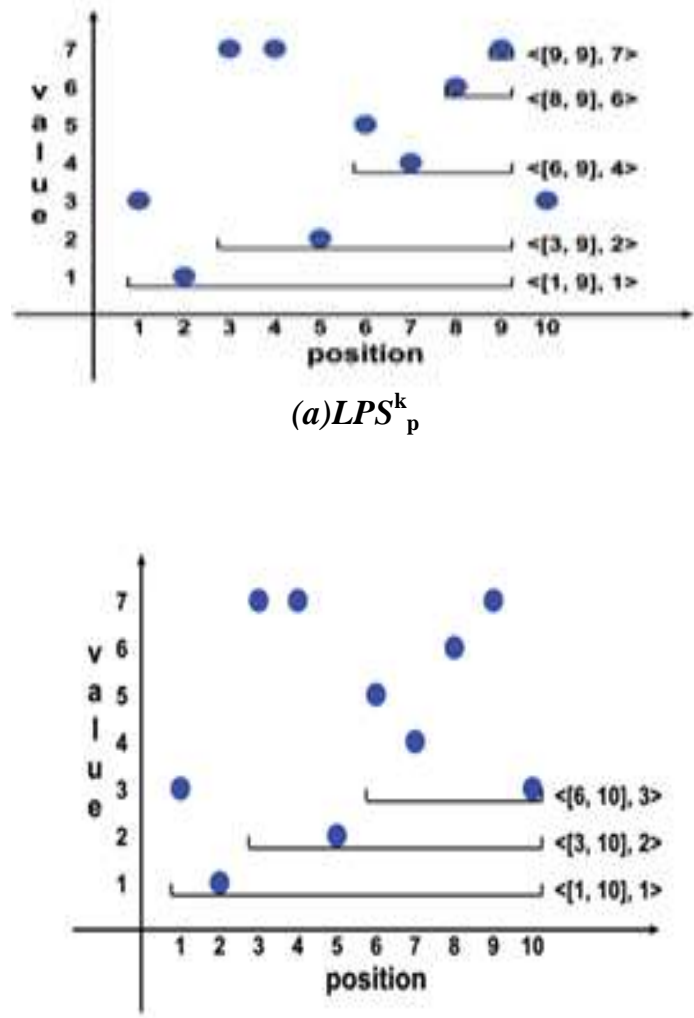

(b) $\mathbf{L P S}_{\text {pk }}^{\mathbf{k}}$

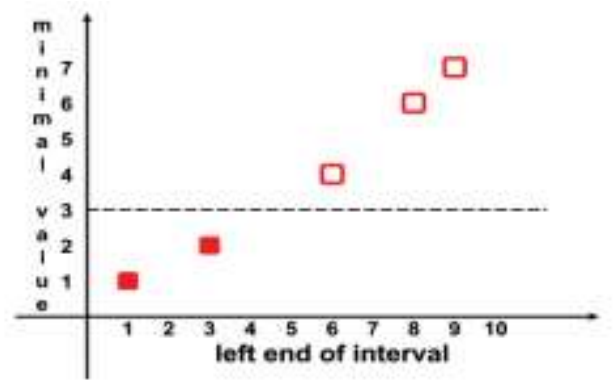

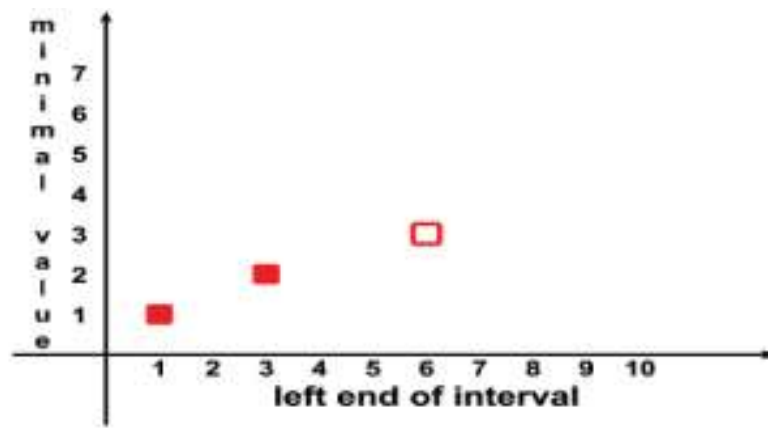

(d) $l-v$ plot of $\mathcal{L P S} \mathcal{S}_{P_{10}}^{10}$

\section{MONITORING PROMINENT STREAKS}

Considered considered one of the right homes of the calculation streak conspicuous disclosure is the capability of looking new statistics phase because the grouping develops always and constantly maintain the traces of unmistakable . As an example, arrange chairmen can look at the traces live in prepare site visitors from internet servers until a selected time. Formally, given the continually developing records arrangement P (eg, statistics glide), adequate-th phase of information that just came signified through pk and request in addition as indicated with the aid of using Pk. Right now, if the purchaser demands PRSP, the lines distending from $\mathrm{Pk}$, we want a talented method to discover them.

As to the pastime of the sky, BST-based totally totally strategy for dynamic update dynamic horizon with traces of new competition and in the long run can be carried out to display the conspicuous lines without adjustment. As to the age of up-and-comers streak, the 1/three approach (gauge, NLPS, LLPs) the usage of 1-skip successive sweep of the statistics association; consequently, they healthy normally into all staring at conditions. Particularly, the new records display pk concerning

\begin{tabular}{|c|c|}
\hline & ORITHM : Continuous Monitoring of Prominent Streaks \\
\hline & iput: The new data entry $p_{k}$ \\
\hline & ompute $\mathcal{L} P S_{p}^{h-1}$ and $\mathcal{L} P \mathcal{S}_{P}^{k}$ by Algorithm 6 \\
\hline & last requested_position $<k-1$ then \\
\hline 3 & for each streak $s$ in $\mathcal{L} P S_{p}^{k-1}$ do \\
\hline 4 & | skyline - shlyine update $($ skyline,s $)$ \\
\hline & $P S_{P_{e}}$ is requested then \\
\hline 6 & for each streak $s$ in $\mathcal{L} P S_{P_{t}}^{b}$ do \\
\hline 7 & | skyline $\leftarrow$ shlyine update(skyline, s) \\
\hline 8 & last requested_position $\leftarrow k$ \\
\hline 9 & // Now, skyline contains all prominent streaks in $P \mathcal{S}_{P_{k}}$ \\
\hline
\end{tabular}

\section{Algorithms}

Given every one of the scenes much of the time, the age rule is straightforward. Calculation 1 depicts how the requirements and their certainty can be decided from the recurrence of scenes. Observe that the gap applied in a calculation to decide the degree of circles and restrictive reasons. 


\section{Calculation 1 .}

Info: a fixed $\mathrm{E}$ of the event kind, occasion grouping s over $\mathrm{E}$, a fixed $\mathrm{E}$ scene, extensive window win, fr min recurrence restrict, and a certainty side min conf. Yield: rules scene proceeds within the s association with a achievement, fr min and min conf

\section{Technique: 1 .}

1. /* discover visit scenes (algorithm 2):

2. */. Compute F (s, wins, minutes fr);

$3 . . / *$ Generate the concepts: */

4. For all $\alpha \in \mathrm{F}$ (s, wins, mins fr) do

5. For all $\beta \prec \alpha$ do

\section{At the off danger that $\operatorname{fr}(\alpha) / \mathrm{fr}(\beta) \geq \min$ conf later}

\section{Yield rule $\beta \rightarrow \alpha$ and consider fr $(\alpha) /$ fr $(\beta)$}

We are currently specializing in following the disclosure task: given an association $\mathrm{s}$ event, a number of $\mathrm{E}$ scene, prevailing huge windows, and the limit min recurrence fr, discover F (s, win, min fr). We supply the foremost detail of calculations and techniques at that point right for its subtasks. We name this method at the entire WINEPI calculation. See section 6 for associated paintings and a few techniques depending on a similar thought.

\section{The fundamental calculation}

Calculation 2 ascertains assortment (s, wins, mins fr) visit scenes of class $\mathrm{E}$ scene. This direct a judicious level calculations (expansiveness first) taking a gander on the accompanying scenes class sub intercourse scene. The search starts offevolved from the maximum extensively diagnosed scenes, ie, scenes with best one occasion. At any price the main calculation computes the upcoming collection of scenes, and in a while have a look at their recurrence of the association of activities. The pressing factor inside the age of up-and-comers gave with the aid of the lemma quick pursues.

Lemma 1. At the off risk that $\alpha$ scene frequently happens in an occasion grouping $\mathrm{s}$, at that factor all sub scene $\alpha \beta^{1}$ go to. Collection incorporates of scenes of up-and-comers is resolved with the aim that each one the little sub scenes often. Those criteria considered secure plum scene that cannot be regularly. An an increasing number of point by factor approach for applicant age and database that pass the degrees are given in the accompanying subsections.

Calculation 2. Information: a hard and fast $\mathrm{E}$ of the occasion kind, the event grouping s over E, a fixed E scene, prevailing wide windows, and the recurrence restrict min Output fr: series F (s, win, min fr) visit scenes. Approach:
1. $\mathrm{C} 1:=\{\alpha \in \mathrm{E}|| \alpha \mid=1\}$
2. $1:=1$;
3. whileCl $6=\varnothing$ do

4. /* Database pass (Algorithms 4 and 5): */5. computeFl $:=\left\{\alpha \in \mathrm{C}_{\mathrm{l}} \mid \mathrm{fr}(\alpha, \mathrm{s}\right.$, win $\left.) \geq \min \mathrm{fr}\right\}$;

6. $1:=1+1$;

7. /* Candidate generation (Algorithm 3): */8. computeC $:=\{\alpha \in \mathrm{E}|| \alpha \mid=1$ and for all $\beta \in \mathrm{E}$ such that $\beta \prec \alpha$ and

9. $|\beta|<1$ we have $\beta \in \mathrm{F}|\beta|\}$;

10. for all 1 do output $F_{1}$;

\section{DISCOVERING GENERAL PROMINENT STREAKS \& RESULTS}

On this segment, we expand the concept of stand immediately and calculations furnished inside the past area for the overall case. Specially, we research the way to locate the top-adequate, multisequence and multidimensional placing strains.

\section{Top-riding good enough Streaks}

Definition five (pinnacle-driving $\mathrm{k}$ Streak). As to the succession $\mathrm{P}=(\mathrm{p} 1, \ldots, \mathrm{pn})$ and the LPS OESD, streak $\mathrm{s} \in$ OESD is putting instantly top-ok if now not commanded by means of okay or extra strains within the OESD - that is $s \in$ OESD and ss pinnacle-ok strains that stand aside are those of LPS ruled by now not precisely different ok LPS, by using definition five. This definition has ramifications. Initially, pinnacle-okay mark that stands need to be domestically sizeable. As an instance, a column is not certified no matter whether it's far simply ruled via one subsuming streak and okay> 1. 2nd, the streak can escape irrespective of whether or now not it is commanded through good enough or distinctive lines greater, for not as a bargain as adequate of the LPS spray portray rule them.

Preserve in thoughts the succession $\mathrm{P}=(20,30,25,30,5$, $5,15,10,15,5)$, as in keeping with the focuses made with the useful resource of the b-ball player in the entirety of its video video games. Streak [3, 4], 25, albeit just dominated thru [2, 4], 25, is a sprint of the ultimate sub and eventually don't stand directly up 2. Natural explanation is that [3, 4], 25 are within the meantime [2,4], 25, and on this way we do not consider it as big. On the other hand, revolutionary [7, 9], 10 are projecting streak on 2. Albeit ruled by using manner of 3 lines, [1, 4], 20, [1, 3], 20, and [2, 4], 25 , stripes command everything of a comparable period and without a doubt one of every three is LPS.

Revolutionary a long term up-and-comer strategies talked about within the past place applies in coming across topokay strains stick out. We actually need more than one minor changes to the pastime of the sky. For LLPs, in mild of the reality that the subsequent applicant ensured to LPS by myself, we clearly want to hold a counter for every factor inside the sky skyline at the same time as this dynamic. Evaluation data the amount of dominators seemingly inside clean achieve. On the point while an applicant is idea about towards the sky now, positioned the skyline at the off risk that it has not as plenty as good enough ruler. A horizon 2nd that erased at the off hazard that it arrives on the counter ok As to the essential techniques and NLPS, they'll expert duce up-and-comers who do now not LPS. An applicant need to 


\section{DISCOVERING NEWSWORTHY THEMES FROM SEQUENCED DATA: A STEP TOWARDS COMPUTATIONAL JOURNALISM}

be reduce if some other competitor push regulations and subsumes it. (study that the two of them object competitors with the right give up of a similar intervening time simultaneously. On this manner, competitors can not be privately commanded by way of factor inside the sky as of now.)

\section{Streaks using Multisequence}

As an example, undergo in mind 3 preparations evaluating to the focuses made with the resource of 3 ball gamers in all recreation-P1 them $=(20,30,25,30$, five, 5 , $15,10,15$, five), $\mathrm{P} 2=(10$, five, $30,35,21,25,5,15,5,25)$, and $\mathrm{P} 3=(5,10,15,5,25,10,20,5,15,10)$. Streak $[1,4]$, 20 of P1 might be P1 mark that hangs out in itself but is ruled through $[3,6], 21$ in $\mathrm{P} 2$. Therefore, no increase of the number one Multisequence streak.The of single-grouping calculation (popular, NLPS, LLPs) for Multisequence simple calculation. We way singular request independently through single-kind calculation and utilizing an ordinary precise sky to hold the strains in their stand. This is, while LPS all collectively Pi identified album, it is contrasted and the prevailing lines inside the sky dynamic, which incorporates traces that stand apart from each one of the adjustments sequences. The of LLPs for checking features showed on the calculation. This calculation can manipulate where the state of affairs of the lines that stick out (up till now) need to be counseled.Take AOL and WC98 for instance. Determine 6 shows the calculation execution time. The x-pivot speaks to the state of affairs of the association, and the $\mathrm{y}$-hub is for $\mathrm{t}$
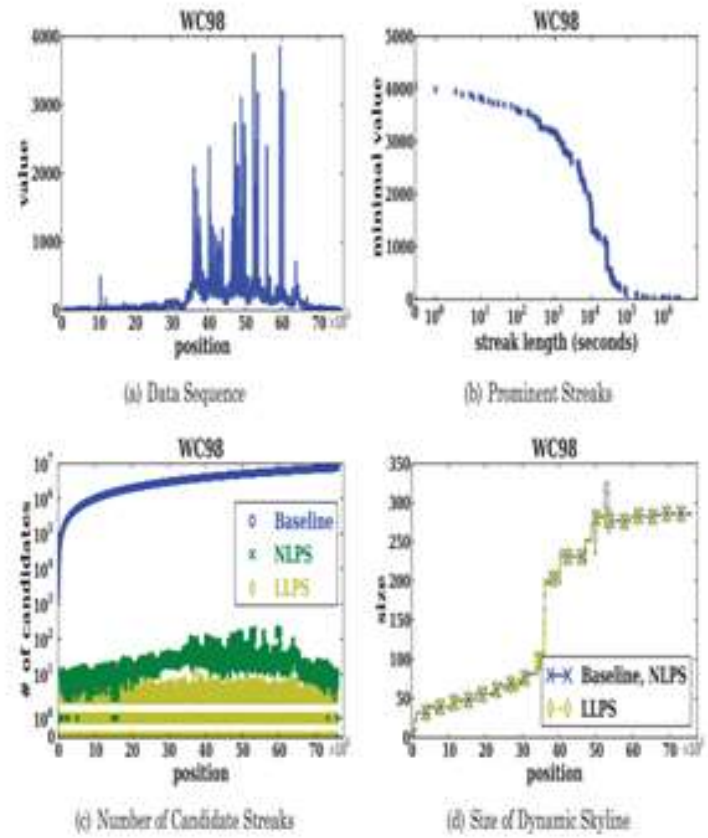

Fig.3 detailed result on WC98, basic streak discovery

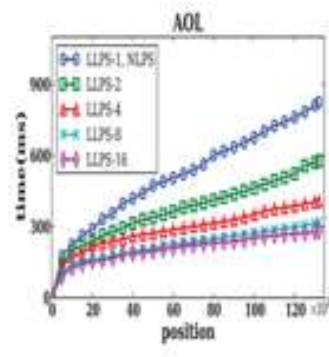

(2) 100

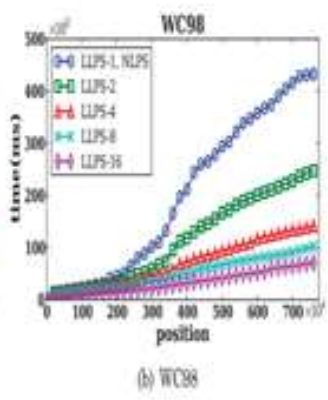

Fig.4 cumulative execution time at various positions for different frequency, basic prominent streak discovery

The recurrence of saying traces that stick out. As an instance, LLPs-1 implies that each time another records section comes, each one of the lines stand apart sofar brought; LLPs-sixteen manner that the traces stand apart are requested at every sixteen statistics passage. As talked about in section 4, LLPs-1 indistinguishable toNLPS (set of rules three), and LLPs-n is indistinguishable toLLPS (algorithm five), in which $\mathrm{n}$ is the grouping length while not developing to any extent in addition. Figures 6 (an) and 6 (b) unmistakably indicates that the execution time thetotal of LLPs-I increment the recurrence of exposing increments (as an example, delivered meantime I diminishes). Figures 7 (an) and 7 (b) further shows how an adjustment in absolute execution time along an change detailing interims. We will see that the execution time drops hastily closer to the start and hastily accomplish near perfect worth in any event, even as the recurrence is adequately excessive (as an instance, brought that widespread traces on each 16 passages.)

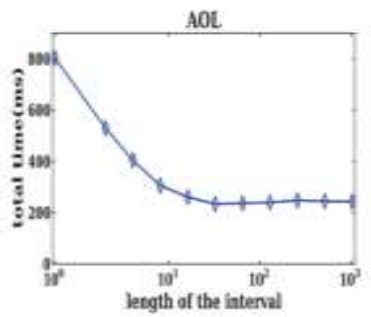

(a) $\mathrm{AOL}$

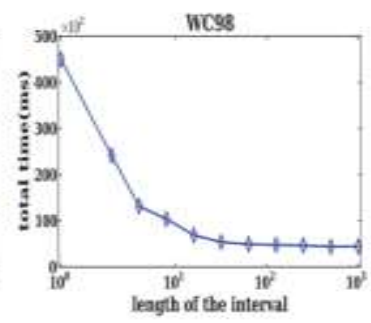

(6) WCys

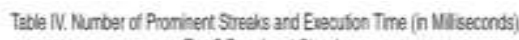
Top-5 Prominet Streaks

\begin{tabular}{|c|c|c|c|c|}
\hline Name & $\begin{array}{c}\text { Promineat Strealks } \\
\text { (t) }\end{array}$ & Raselize & XIPS & LWFS \\
\hline Gedi & $14 \pi$ & 1854 & 348 & 44 \\
\hline Fiver & 14 & $6.81 \times 10^{4}$ & 255 & 57 \\
\hline Melb1 & 150 & $6.6 \times 10^{7}$ & 572 & 96 \\
\hline Meltes & 160 & $301 \times 10^{2}$ & 445 & 150 \\
\hline Wine & 131 & $3.68 \times 10^{\circ}$ & 1329 & 140 \\
\hline Wili:2 & 115 & $1.58 \times 10^{7}$ & 365 & 172 \\
\hline Wile? & 172 & $1.5 \times 10^{\frac{7}{7}}$ & 473 & 136 \\
\hline S\$500 & 526 & $7.09 \times 10^{6}$ & 13,200 & 270 \\
\hline $\mathrm{HPQ}$ & 251 & $>$ Whear & 3211 & 178 \\
\hline IEM & 212 & $>$ W beens & 5914 & 229 \\
\hline AOL & 20 & 310 heus & 25,000 & 258 \\
\hline WCS5 & 92 & $>$ thbers & $>10$ bours & 12,300 \\
\hline
\end{tabular}


Experimental Results on General Prominent Streak Discovery

On this area, we speak approximately the aftereffects of the above-ok, multisequence, and multidimensional revelation jutting streak. Toward the stop of this vicinity, we likewise gift the aftereffects of trials that have fused an alternate expansion.

\section{Pinnacle-riding okay Streaks}

Investigations on the pinnacle-k conspicuous lines accomplished at the equal dataset are mentioned in phase 6.1. For each dataset, table IV shows the amount of pinnacle-5 conspicuous strains (eg, KPSP in Definition 5) and a benchmark calculation execution time, NLPS and LLPs broadened. Observe that the quantity of strains appeared in table II applicants maintain as in advance than, as an instance, the approach for progressive a long time applied a similar opportunity for the top-adequate the use of spray portray, as pointed out in segment five.1.

As desk IV appears, contrasted with the hour of execution in desk III (ie, when locating the primary 1 squiggle stand), time of utilization of the benchmark elevated by at least one devices of quantity, while the presentation of NLPS and LLPs are corrupted by the usage of short of what one request for greatness by means of and huge, this is clarified as pursues. Coming across pinnacle-ok conspicuous spray portray brings about extra prices of jogging the sky than to discover a excellent 1 champion spray painting. All of the more explicitly, the operating charge is controlled by means of the use of the sum horizon predominance correlations among up-and-comer traces and functions in a powerful sky. In this manner, the quantity of examinations increments each the quantity of strains and the dimensions of the sky dynamic competitor.

\section{Ends AND destiny paintings}

We added a shape for locating non-stop scenes within the information grouping. The form incorporates of characterizing scene as quite asked association of events, and examine the window all collectively. We depict a calculation,

\section{CONCLUSION}

In this article, we take a look at the difficulty of finding the strains continue to be within the succession information. An unmistakable streak is long once more to lower back subsequence includes of developments is honestly awesome (little). We recommend a green approach based

At the idea of LPS. We display that the lines stand aside is a chunk of LPS and that the diploma of LPS not exactly the period of the association information. LPS direct primarily based absolutely strategy we guarantee to bear in mind truely the LPS, on this manner mission a noteworthy decrease in stripes applicants. The individual and the proposed calculation is additionally reached out to find out normal pinnacle-ok, multisequence and multidimensional placing strains. The trial effects for some real datasets checked the viability of the proposed technique..

\section{REFERENCES}

1. S. Laxman, P. Sastry, good enough. Unnikrishnan, "A brief calculation for finding incessant scenes in occasion circulation", Proc. Thirteen ACM SIGKDD Int. Conf. Knowl. The revelation of facts Mining, pp. 410-419, 2007.

2. G. Zhang, X. Jiang, P. Luo, M. Wang, C. Li, "discovering everyday unmistakable strains inside the request for statistics", ACM Trans. Knowl. Records revelation, vol. 8, no. 2, 2014.

3. R. Agrawal, S. Gollapudi, A. Halverson, S. Ieong, "Enhancement of listed lists", Proc. 2 ACM Int. Conf. Net seek facts Mining, pp. Five-14, 2009.

4. Brants T., F. Chen, A. Farahat, "A framework for the recognition of each other occasion", Proc. 26 Annu. Int. ACM SIGIR Conf. Res. Create. Informaion Retrieval, pp. 330-337 2003.

5. M. D. Berg, O. Cheong, M. V. Kreveld, M. Overmars, Computational Geometry: Algorithms and applications, Santa Clara, CA, the us: Springer-Verlag, 2008.

6. L. Golab, H. Karloff, F. Korn, A. Saha, D. Srivastava, "Consecutive conditions", Proc. VLDB Endowment, vol. 2, no. 1, pp. 574-585, 2009.

7. k. Edwards, S. Griffiths, W. S. Kennedy, "Fractional arrangements of hose close-exchange off amongst adaptability and optimality" in Approximation Randomization and Combinatorial Optimization. Calculations and techniques, Berlin, Germany: Springer, pp one hundred ten-a hundred twenty five, 2013 ..

8. N. Alon, B. Awerbuch, Y. Azar, "the internet set spread hassle", Proc. 35 Annu. ACM Symp. Speculation Comput., Pg. One hundred-one hundred and five 2003. 